\title{
In Silico Analysis of Inhibiting Papain-like Protease from SARS-CoV-2 by Using Plant-Derived Peptides
}

\author{
Mohammad Moradi ${ }^{1,2} \cdot$ Reza Golmohammadi $^{1} \cdot$ Ali Najafi $^{1} \cdot$ Mehrdad Moosazadeh Moghaddam $^{3}$. \\ Mahdi Fasihi-Ramandi ${ }^{1} \cdot$ Reza Mirnejad $^{1}$
}

Accepted: 21 October 2021 / Published online: 9 December 2021

(c) The Author(s), under exclusive licence to Springer Nature B.V. 2021

\begin{abstract}
SARS-CoV-2 is a corona virus that has been the cause for one of the deadliest pandemics of history, started since 2019. Suppressing the activity of the critical enzymes in the SARS-CoV-2 could potentially inhibit a vital step in viral life cycle. Papain-like protease (PLpro) could be regarded as a critical enzyme in viral replication of SARS-CoV-2. In this research, it was aimed to suppress the activity of PLpro enzyme by using potential plant-derived protease inhibitor peptides. For this purpose, 11 plant derived peptides that could potentially inhibit protease activity were selected from literature. The structures of the PLpro and the peptide ligands were acquired from PDB (protein data bank) and after structural optimization, were docked by using HADDOCK 2.4 program. Analyzing the results indicated that VcTI from Veronica hederifolia provides effective molecular interactions at both liable $\mathrm{Zn}$ site and classic active site of PLpro, making it a potential inhibitory ligand for this enzyme that could be used for halting the replication of SARS-CoV-2. Molecular dynamic assay confirmed that the selected receptor and ligand complex was stable. Future in vitro and in vivo investigations are required to verify the efficiency of this compound as a potential therapeutic against SARS-CoV-2 infection.
\end{abstract}

Keywords Plant-derived peptides $\cdot$ In silico $\cdot$ SARS-CoV-2 $\cdot$ Papain-like protease

\section{Introduction}

In 2019 , a new member of corona viride family called SARS-CoV-2, caused one of the worst pandemics of history that infected a considerable number of people, resulting a catastrophic condition in the modern medicine (Ram et al. 2020). Further, SARS-CoV-2 was transferred to the people from various countries, via different roots such as travelers, and it resulted in a pandemic condition that was announced by WHO (Malik et al. 2020). The major sign of infection with this new member of corona viride family (also called COVID-19) is the pneumonia like symptom, but other signs

Reza Mirnejad

rmirnejad@bmsu.ac.ir

1 Molecular Biology Research Center, Systems Biology and Poisonings Institute, Baqiyatallah University of Medical Sciences, Tehran, Iran

2 Department of Biotechnology, Faculty of Biological Science and Technology, University of Isfahan, Isfahan, Iran

3 Applied Biotechnology Research Center, Baqiyatallah University of Medical Sciences, Tehran, Iran such as headache, fatigue and loss of taste have also been reported (Ram et al. 2020).

To present an effective therapeutic against SARS-CoV-2, having a conclusive knowledge about the viral structures is necessary (Mohabatkar et al. 2021a, 2021b; Valle et al. 2020), which could enable to find a more appropriate candidates for targeting. Regarding the potential target for COVID-19 drug development, some molecular structures have been suggested including spike glycoprotein, main protease, and papain-like protease (Wong et al. 2021a, 2021b). Suppressing these components or their activity could potentially regress the malady condition of COVID-19 (Amin et al. 2021).

SARS-CoV-2 infection is linked with the life cycle of this virus, which includes its attachment to the target cells, entering to the cell, gene replication, transcription of the RNA, and translation of viral proteins that causes maturation of viral structure and its release from the infected cell. By suppressing each part of this cycle, the invasion of virus to the host cells could be stopped, and it could be helpful to improve the condition of infected individuals (Ryu 2017). 
Proteases are necessary components of the SARS-CoV-2 life cycle. Upon the entry of SARS-CoV-2 into its target cells, this virus expresses two cysteine protease enzymes; named 3-chymotrypsin-like protease and papain-like protease which are very necessary for the replication of this pathogen (Gao et al. 2021). Due to having such an important role, these proteases could be regarded as potential molecular targets for studying therapeutics against COVID-19 (Báez-Santos 2015).

Crystallography structure analysis of SARS-CoV-2 PLpro has demonstrated that this enzyme has three main active domains, which could have an important effect in the damages caused by COVID-19, including a main catalytic site, a Zn-binding domain which is necessary for $3 \mathrm{D}$ structure of the virus, and a ubiquitination domain (Rut et al. 2020). Each of these domains could be considered as potential targets for developing therapeutic agents. At the current research, we decided to stay focused on the $\mathrm{Zn}$ site (Cys189-X-X-Cys192-Xn-Cys224-X-Cys226) and the catalytic site (Cys111-His272-Asp286) which are more highlighted targets for drugs development (Maiti 2020). At this study, we aimed to target these domains by plant-derived peptides.

In this enzyme, the conserved residues of Cys are necessary for proper binding of $\mathrm{Zn}^{2+}$ which is critical for appropriate folding of protein. Moreover, they have a catalytic role by nucleophilic reaction on the substrate, which helps the appropriate functioning of this enzyme. Considering this fact, blocking the $\mathrm{Zn}$ site and/or stopping the Cys amino acids at the main catalytic domain of this viral enzyme could be the potential antiviral therapies, that prevent the proper function of PLpro and halt the cycle of life for SARSCoV-2 (Ibrahim et al. 2020; Nejat \& Sadr 2019; Osipiuk et al. 2021).

So far, there is no universally accepted therapeutic agent for targeting SARS-CoV-2 PLpro, accordingly any study that could increase the knowledge about targeting this vital protein would be useful for developing new agents against this virus. Accordingly, the first stage of the study is to identify potential protease inhibitors that could effectively bind to the conserved Cys amino acids of PLpro enzyme (Mitra et al. 2021; Sargsyan et al. 2020). Keeping this fact in mind, we decided to investigate the efficacy of potential plant-derived peptide protease inhibitors for blocking the two critical sites for $\mathrm{Zn}$ interaction site and the classic active site of PLpro of SARS-CoV-2.

Until now, more than 6700 plant-derived proteinaceous protease inhibitors (PIs) have been reported. These peptides are categorized into various families based on their structural similarity and sequence homology including Phytocystatins (Farady \& Craik 2010), Serpins (Gomes 2011), Kunitz-type protease inhibitors (Bateman 2011), etc. So far, the total number of 104 plant families are known to produce
PIs, with differences in the diversity (Hellinger \& Gruber 2019).

One of the most highlighted field that could be useful for investigating the efficacy of drugs against various disorders, including COVID-19, is the field of bioinformatics (Nabati et al. 2020). Application of the computer tests before taking them to the in vitro and in vivo level can diminish the financial burden and the use of experts' time and energy, along with reducing the need for animal sacrifice (Haghighi 2021; Mohabatkar, Ebrahimi, \& Moradi 2021a, b). Bioinformatics method provide an in silico platform for scientist through which they could investigate the efficiency of thousands of potential therapeutic reagents by working on their computers (Ahmadi et al. 2021; Gharbavi et al. 2020). So far, bioinformatics methods have been used for developing recombinant vaccines and investigating plenty of natural and synthetic reagents against COVID-19 (Behmard et al. 2020; Fallah et al. 2021).

At this research study, it was planned to examine the efficacy of potential plant-derived PIs peptides against SARS-CoV-2 as antimicrobial agents via bioinformatics investigation. Accordingly, we analyzed the potential of 11 peptide-based PIs to suppress the CoV-2 PLpro using in silico methods.

\section{Material and Methods}

\section{Data Collection}

\section{Protein Receptor}

The structure of SARS-CoV-2 Papain-like protease was fetched from PDB (6WUU) with a resolution of $2.79 \AA$, provided through $\mathrm{X}$-Ray differentiation technique by Rut et al. (Rut et al. 2020). Chimera tool was used for removing the extra ligands from the selected PDB file of the target protein and its energy minimization for further molecular docking assay (Pettersen et al. 2004).

\section{Peptide Ligands}

The potential plant-derived peptides for suppressing the protease enzyme of COVID-19 were selected from literature and their structures were fetched from protein data bank (PDB). The criteria for choosing these peptides was their potential to act as PIs and we tried to study peptides from different families of inhibitors. Only the peptides with small size that could enter human cell and are none-toxic which had been suggested suitable for human use were selected from previous studies (Hellinger \& Gruber 2019).

These peptides include Zea mays-derived Hageman factor (1BEA) as a member of $\alpha$-Amylase inhibitors and 
bifunctional trypsin inhibitors (Behnke et al. 1998), Oryza sativa-derived oryzacystatin-I (1EQK) as a member of Phytocystatins (Nagata et al. 2000), Solanum tuberosum-derived potato CPI (1H20) as a member of Metallocarboxypeptidase inhibitors (González et al. 2003), Oldenlandia affinis-derived kalata B1 from (1NB1) as a member of Cyclic cysteinerich peptides (Rosengren et al. 2003), TI-II from Solanum lycopersicum-derived (1PJU) (Barrette-Ng et al. 2003) and Cucurbita maxima-derived CMTI-V (1TIN) as members of Potato-type inhibitors (Cai et al. 1995), A. thaliana-derived mustard-TI from (1JXC) as a member of Mustard-type trypsin inhibitors (Zhao et al. 2002), Ecballium elateriumderived EETI-II (2IT7) as a member of Squash trypsin inhibitors and cyclic Momordica-type trypsin inhibitors (Heitz et al. 2008), Veronica hederifolia-derived VcTI (2PLX) as a member of $\alpha$-Hairpinin inhibitors (Conners et al. 2007), Glycine max-derived soybean BBI (5J4Q) as a member of Bowman-Birk inhibitors family (Tornøe et al. 2017), Cicer arietinum-derived Kunitz inhibitor (5XOZ) from Kunitztype protease inhibitors family (Bendre et al. 2019). Chimera tool was again used for removing the extra ligands from the PDB file of the peptide constructs and optimizing them for further molecular docking assay (Pettersen et al. 2004).

\section{Molecular Interactions Assay}

Molecular docking is an effective tool for investigating interactions between biological receptors and their ligands which provides information regarding the formed interactions types, and the energy of the complex (Haghighi et al. 2020; Nabati et al. 2020). To study the molecular interactions between the potential proteases inhibitors from plants, molecular docking was performed between the selected peptides in complex with liable $\mathrm{Zn}$ site and the classic active site of COVID-19 PLpro enzyme. Molecular docking studies were performed by HADDOCK 2.4 server (available at https:// wenmr.science.uu.nl/haddock2.4/) (Van Zundert et al. 2016), using the following setting:

Regarding distance restraints; force constant for center of mass contact restraints was fixed on one, force constant for surface contact restraints was set on one. Number of partitions for random exclusion was two, and Radius of gyration was fixed on 17.78 value.

Regarding the sample parameters; number of structures for rigid body docking was set on 1000, the count of trials for rigid body minimization was five, and 200 structures were set for semi-flexible refinement. 200 constructs were set for final refinement and analysis. Fraction of Common Contacts (FCC) method was applied for clustering the results of docking with 0.6 RMSD cutoff and minimum clustering size was set on four.

At the analysis parameters, Cutoff distance (protonacceptor) of 3.5 was selected to define a hydrogen bond and cutoff distance (carbon-carbon) of 3.9 was chosen to define a hydrophobic contact.

\section{Complex Analysis}

For analyzing the interactions of ligand and receptors after molecular docking, the complex was studied by discovery studio visualizer (Pawar \& Rohane 2021) to investigate the surface of interactions between the selected ligands and PLpro enzyme structure. PDBsum tool (available at: http:// www.ebi.ac.uk/thornton-srv/databases/cgi-bin/pdbsum) (Laskowski et al. 2018) was used for checking the details of amino acids interactions and visual presenting the interacting residues.

The stability of the selected ligand and PLpro enzyme complex was investigated using the molecular dynamics simulation assay by version 5.0 of GROMACS program (Hess et al. 2008; Oostenbrink et al. 2004). For this simulation assay, all-atom OPLS force field was used for analyzing the formed complex stability and a cubic box was defined as the unit cell. Spc216 was used as a generic equilibrated 3-point solvent model for this investigation.

Genion tool was used as the tool for adding ions in GROMACS program to replace the water molecules with specified ions. Grompp was used for processing coordinate file and topology in order to create an atomic-level input. After performing energy minimization with $\mathrm{F}_{\max }$ of no greater than $1000 \mathrm{~kJ} \mathrm{~mol}^{-1} \mathrm{~nm}^{-1}$, system was exposed to two steps of equilibration, including $N V T$ (constant Number of particles, Volume, and Temperature) and NPT (Number of particles, Pressure, and Temperature) each step for a time of 100 ps. Afterward, the MD run was performed in $20 \mathrm{~ns}$ for investigating the stability of the complex. Qtgrace version 0.2.6 for windows was used to visualize the resultant analysis graphs.

\section{Results}

\section{Molecular Interactions of Zn Site}

The results of molecular docking of $\mathrm{Zn}$ liable site from PLpro with the selected plant-derived peptides by HADDOC server indicated that VcTI compound (2PLX) provides the highest docking score of -76.1 , with RMSD of 0.4 and $\mathrm{Z}$-score of -2.2 . HADDOCK clustered 115 complexes in 18 clusters, which represents $57 \%$ of the water-refined models HADDOCK created (Fig. 1S). Cluster 2 with the size of 10 showed to provide the most reliable results for this docking assay (Fig. 2S).

Complex analysis indicated that 26 residues from the enzyme and 18 residues of the VcTI compound were involved at the molecular interactions. In the receptor-ligand 

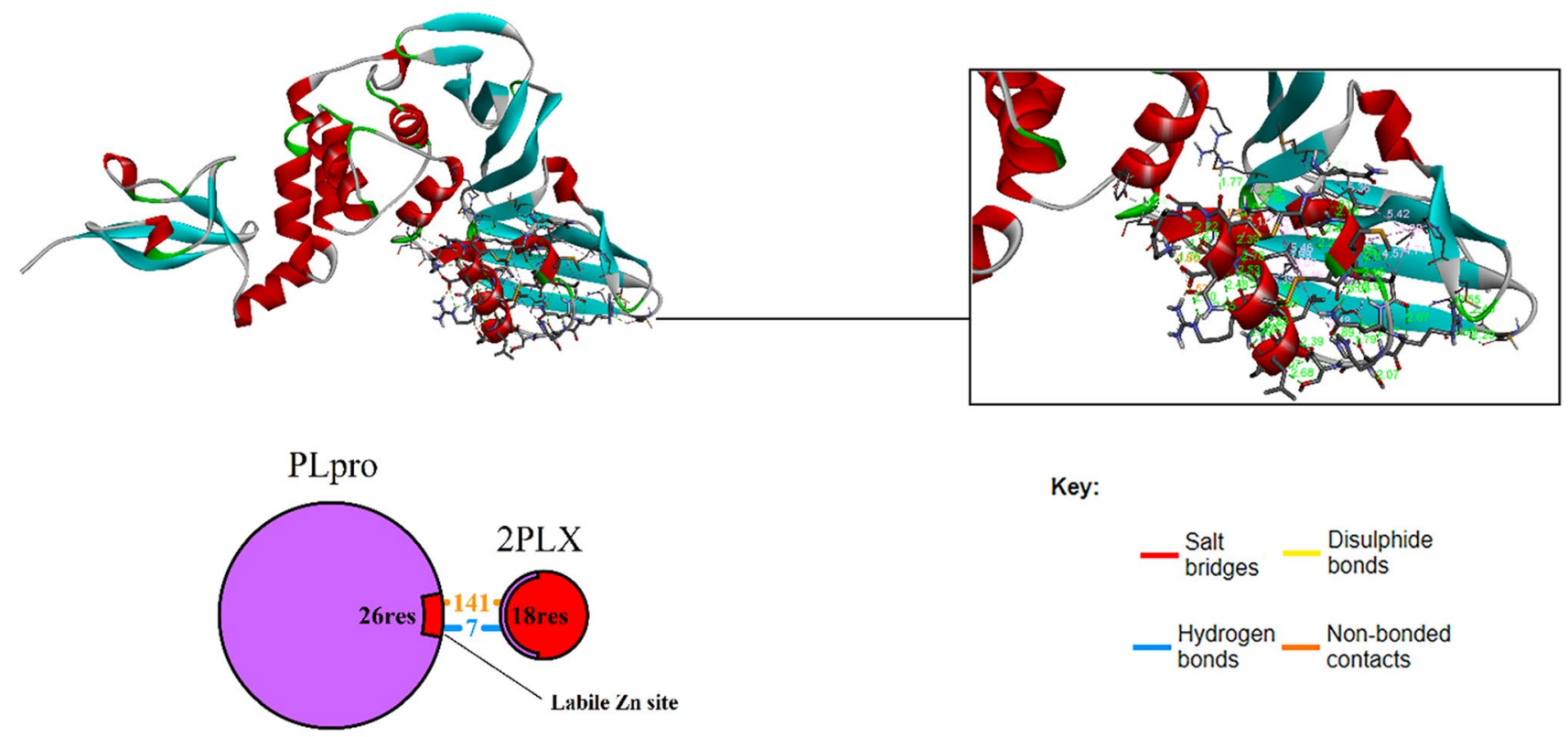

Key:
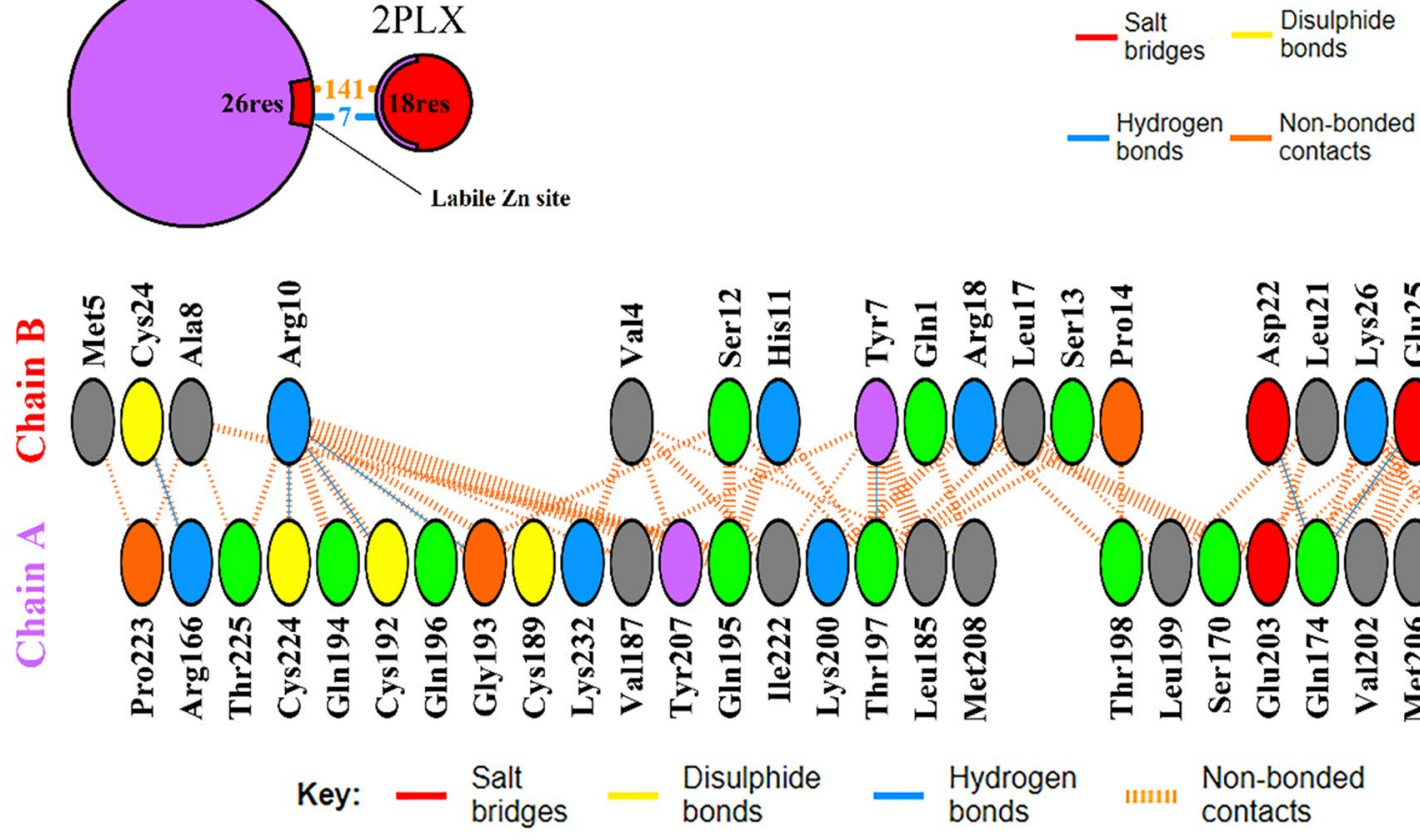

Residue colours: Positive ( $(H, K, R)$; negative $(D, E)$; $S, T, N, Q=$ neutral; $A, V, L, I, M=$ aliphatic; $F, Y, W=$ aromatic; P, G = Pro\& Gly;

Fig. 1 The molecular interactions between VcTI and classic site of PLpro enzyme. Total of 148 interactions were detected among which seven were hydrogen bonds and 141 were electrostatics interactions.

complex, 141 non-bound interactions and 14 hydrogen bonds were found. The inspection of the interacting residues indicated that three of the critical Cys residues were involved in the interaction with the VcTI compound. In the enzyme site, both Cys 192 and Cys224 were bounded to the Arg10 of VcTI via hydrogen bonds, and Cys189 had non-bound interaction with this residue; therefore, $\operatorname{Arg} 10$ of VcTI is a very important residue for suppressing PLpro activity (Fig. 1). For more details about the receptor surfaces of interactions with potential inhibitors, please refer to (Fig. 2).

From the other compounds, Zea mays-derived Hageman factor (1BEA) provided the highest Vander Waals energy of $-100.7 \mathrm{kcal} / \mathrm{mol}$, which makes it another potential effective
Two of the key Cys residues (192, and 224) were involved in hydrogen bonds with Arg10 of VcTI peptide

candidate, but its docking score was -26.2 . Table 1 provides more detail of the interactions between selected plantderived peptides as potential inhibitors interactions with $\mathrm{Zn}$ liable site of PLpro enzyme.

\section{Molecular Interactions with Main Catalytic Site}

The results of molecular docking the classic active site from PLpro with selected plant-derived peptides by HADDOC server showed that similar to the liable $\mathrm{Zn}$ site, VcTI provides the highest docking score of -104.7 , with RMSD of 0.3 and Z-score of -2.2 at the classic active site. HADDOCK clustered 170 structures in eight clusters, 

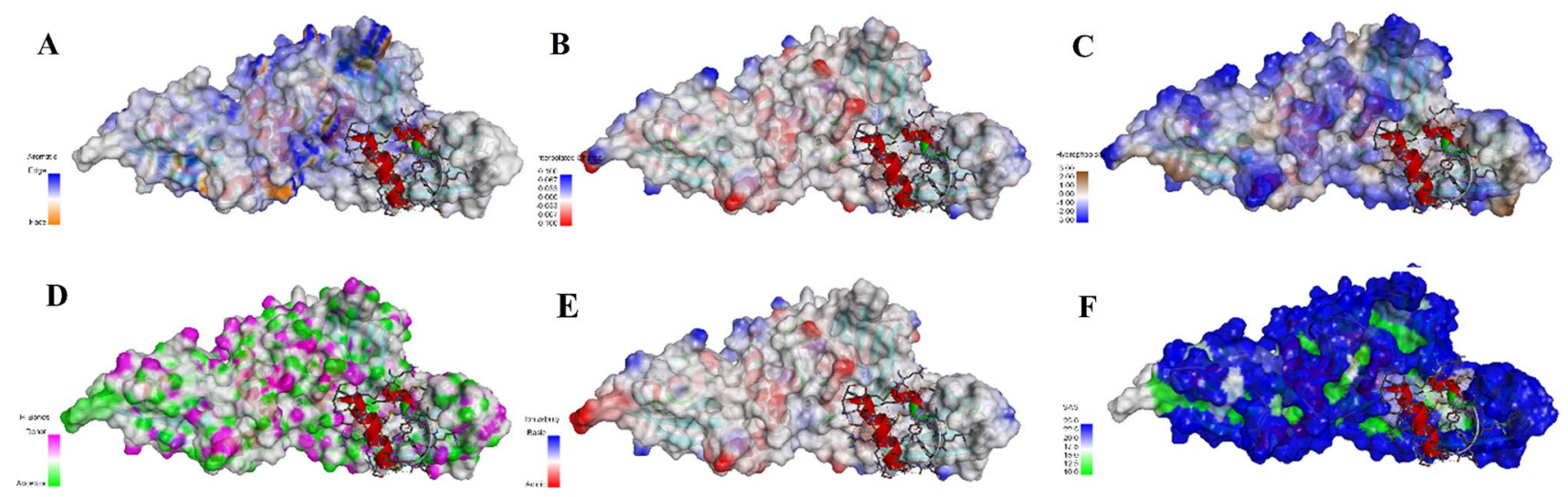

Fig. 2 VcTI receptor surface interactions with liable Zn site of PLpro enzyme with respect to surfaces of A. Aromatic interactions B. Interpolated charges C. Hydrophobicity D. Hydrogen bonds E. Ionizablity and F. Solvent accessibility

Table 1 Results of molecular interaction analysis between selected plant-derived peptides and Zn site from papain-like protease of COVID-19

\begin{tabular}{|c|c|c|c|c|c|c|c|c|c|}
\hline $\begin{array}{l}\text { Peptide PDB } \\
\text { code }\end{array}$ & $\begin{array}{l}\text { HADDOCK } \\
\text { score }\end{array}$ & Cluster size & $\begin{array}{l}\text { RMSD from the } \\
\text { overall lowest } \\
\text { structure }\end{array}$ & $\begin{array}{l}\text { Vander } \\
\text { Waals } \\
\text { energy }\end{array}$ & $\begin{array}{l}\text { Electro- } \\
\text { statics } \\
\text { energy }\end{array}$ & $\begin{array}{l}\text { Desol- } \\
\text { vation } \\
\text { energy }\end{array}$ & $\begin{array}{l}\text { Restraint } \\
\text { violation } \\
\text { energy }\end{array}$ & $\begin{array}{l}\text { Buried } \\
\text { surface } \\
\text { area }\end{array}$ & Z-score \\
\hline 1BEA & -26.2 & 5 & 0.7 & -100.7 & -242.7 & -17.6 & 1406.2 & 3123 & -1.7 \\
\hline $1 \mathrm{EQK}$ & -6.3 & 12 & 9.9 & -90.1 & -124.4 & -8 & 1167 & 3092.5 & -1.8 \\
\hline $1 \mathrm{H} 20$ & -51.9 & 15 & 4.3 & -51.2 & -167.3 & -13.2 & 460.4 & 1683.2 & -1.8 \\
\hline 1NB1 & -44.9 & 8 & 0.8 & -53.1 & -102.1 & -0.2 & 288.7 & 1546 & -1.9 \\
\hline 1PJU & -44.8 & 5 & 0.6 & -96.9 & -327.7 & -8.5 & 1260.7 & 3123.4 & -2.4 \\
\hline 1TIN & -32.2 & 18 & 9.1 & -68.4 & -260.9 & 10.2 & 782.4 & 2354.4 & -1.5 \\
\hline $1 \mathrm{JXC}$ & -55.7 & 6 & 1.5 & -70.1 & -289.4 & -9.6 & 818.9 & 2324.3 & -1.6 \\
\hline 2IT7 & -72.8 & 19 & 0.4 & -50.3 & -279.9 & 8.9 & 246.2 & 1901.8 & -2.7 \\
\hline $2 \mathrm{PLX}$ & -76.1 & 10 & 0.4 & -75.1 & -134.3 & -0.3 & 261.2 & 2044.9 & -2.2 \\
\hline $5 J 4 Q$ & -29.9 & 6 & 8.7 & -62 & -275.9 & 5.6 & 817.6 & 2356.8 & -1.2 \\
\hline $5 \mathrm{XOZ}$ & 92.4 & 11 & 1.7 & -72.6 & -415.1 & 14.5 & 2335.4 & 2702.2 & -1.5 \\
\hline
\end{tabular}

representing that $85 \%$ of the water-refined models HADDOCK were generated (Fig. 3S). Cluster 2 with the size of 58 showed to provide the most reliable results for this docking assay (Fig. 4S).

18 residues from the enzyme and 16 residues of the VcTI compound were involved in the interaction. In the receptor-ligand complex, 124 non-bound interactions, three salt bridges and 7 hydrogen bonds were found (Fig. 3). Regarding the PLpro enzyme classic site surface of interactions complexed with VcTI please refer to (Fig. 4).

The inspection of the interacting residues indicated that all of the three critical residues in active site were involved in the interaction with the VcTI compound. In the classic active site of the enzyme, Asp286 provided a hydrogen bond with Lys 3 of the peptide, and the other to catalytic residues had non-bound interactions with PLpro classic active. Arg10 of peptide was again involved in two hydrogen bonds and a salt bridge with Gln161 of the enzyme, and a non-bound interaction with Asn109, showing an important role in the complex formation.

From the other compounds, TI-II from Solanum lycopersicum-derived (1PJU) provided the highest Vander Waals energy of $-85.5 \mathrm{kcal} / \mathrm{mole}$, which makes it another potential effective candidate, but its docking score was 3.3, indicating a lower shape complementary score. For more details about the selected plant derived peptides interactions with the classic active site of PLpro, please refer to Table 2.

Since the most of inhibitory effect of the VcTI is expected to be via inhibiting the main catalytic site and this complex had a higher docking score than $\mathrm{Zn}$ site, the molecular dynamics simulation for this site was performed in a 20 ns. In this study, RMSD (root-mean-square deviation) which is a standard measure of structural distance between coordinates was investigated as an important element of the stability of the complex conformation. RMSD 

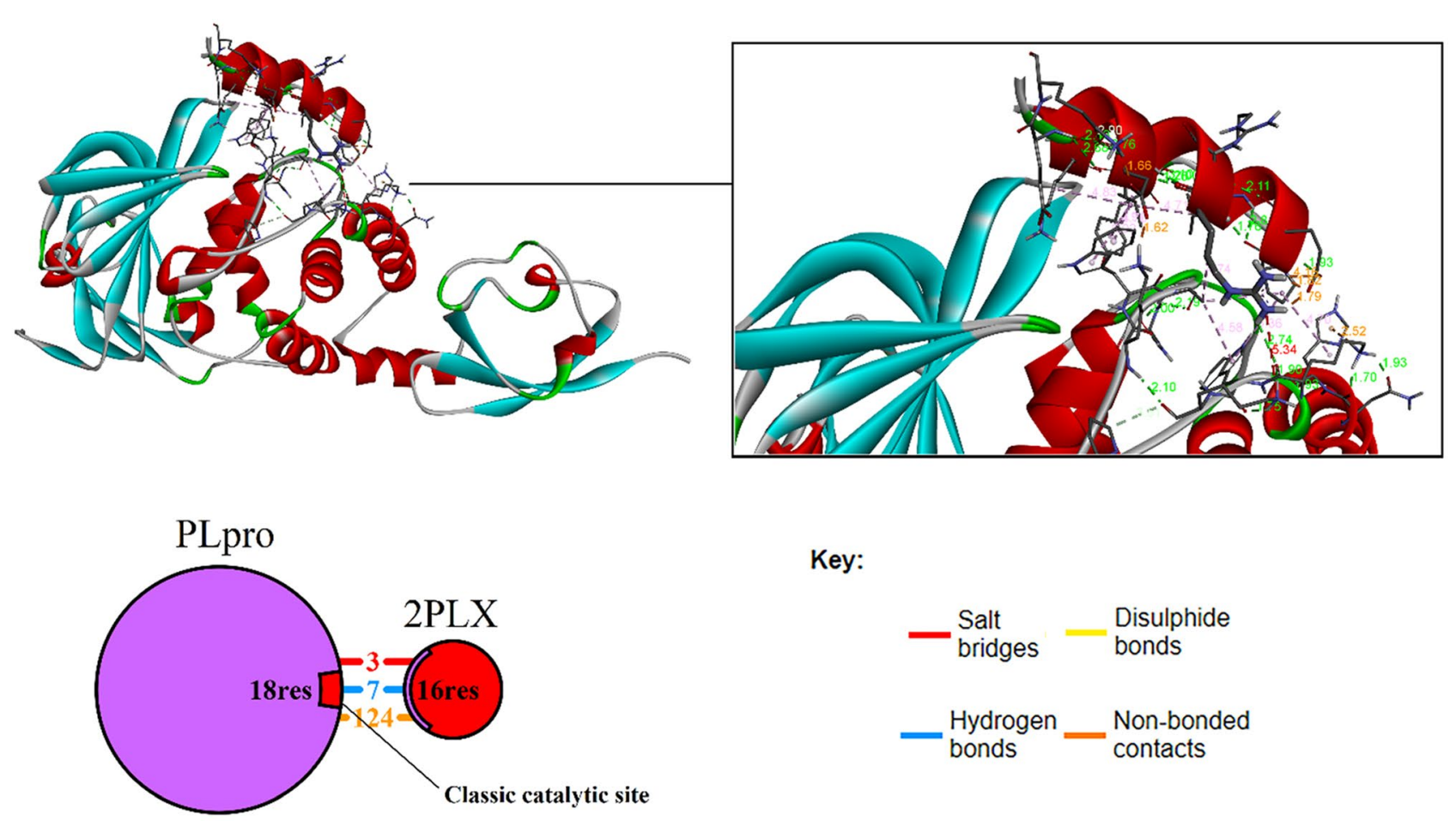

Key:
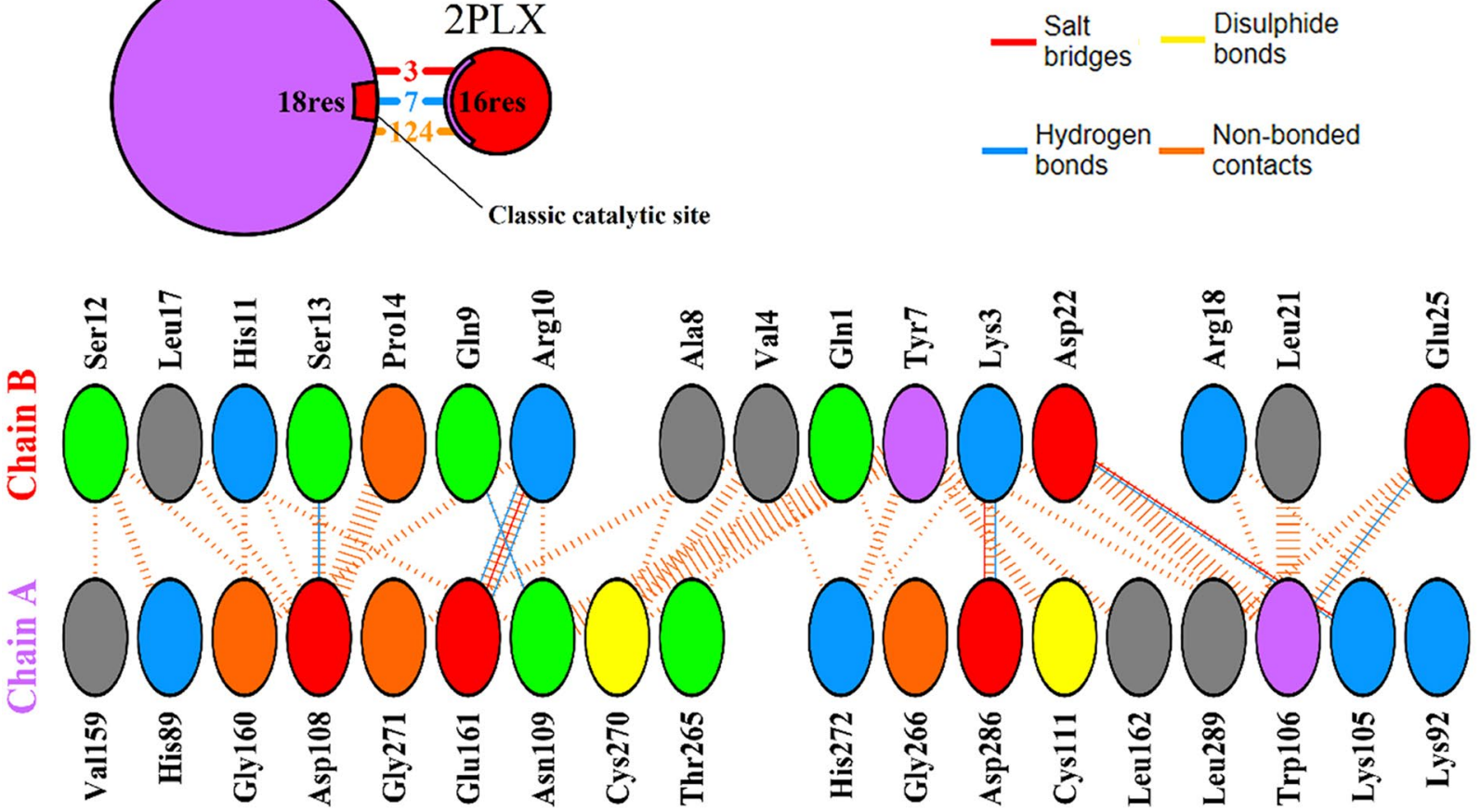

Key: - $\begin{aligned} & \text { Salt } \\ & \text { bridges }\end{aligned} \quad \begin{aligned} & \text { Disulphide } \\ & \text { bonds }\end{aligned} \quad \begin{aligned} & \text { Hydrogen } \\ & \text { bonds }\end{aligned} \ldots \ldots \begin{aligned} & \text { Non-bonded } \\ & \text { contacts }\end{aligned}$

Residue colours: Positive $(H, K, R)$; negative $(D, E) ; S, T, N, Q=$ neutral; $A, V, L, I, M=$ aliphatic; $F, Y, W=$ aromatic; P, G = Pro\&Gly;

Fig. 3 The molecular interactions between VcTI peptide and the classic active site of PLpro enzyme. Total 134 interactions, including salt bridge, hydrogen bonds and non-bonded contacts were indicated. All

related to equilibrated and crystal structure of the complex was scrutinized in this study, too. The minor changes of RMSD indicates (approximately $0.15 \mathrm{~nm}$ ) indicates that of the three key residues of the active site (Cys111-His272-Asp286) were involved in the interactions with the potential inhibitor ligand

this complex has stability over the selected time period of analysis.

Moreover, Radius of gyration ( $\mathrm{Rg})$ of a protein which is a measure of its compactness, indicated that the complex 

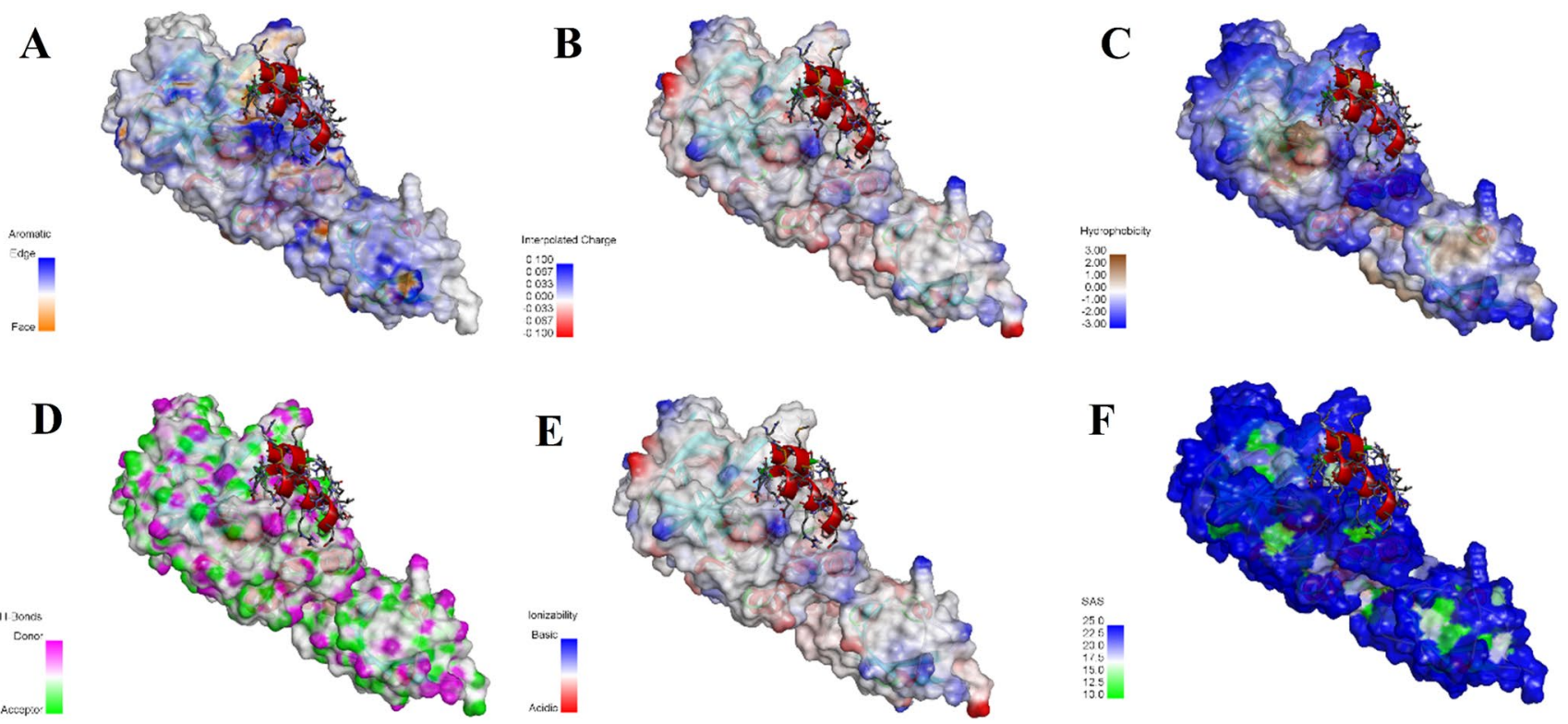

Fig. 4 PLpro enzyme classic site surface of interactions complexed with VcTI regarding to A. Aromatic interactions B. Interpolated charges $\mathbf{C}$. Hydrophobicity D. Hydrogen bonds E. Ionizablity and F. Solvent accessibility

Table 2 Molecular interactions of plant-derived peptides with classic catalytic site of PLpro enzyme

\begin{tabular}{|c|c|c|c|c|c|c|c|c|c|}
\hline $\begin{array}{l}\text { Peptide } \\
\text { PDB code }\end{array}$ & HADDOCK score & Cluster size & $\begin{array}{l}\text { RMSD from the } \\
\text { overall lowest } \\
\text { structure }\end{array}$ & $\begin{array}{l}\text { Vander } \\
\text { Waals } \\
\text { energy }\end{array}$ & $\begin{array}{l}\text { Electro- } \\
\text { statics } \\
\text { energy }\end{array}$ & $\begin{array}{l}\text { Desol- } \\
\text { vation } \\
\text { energy }\end{array}$ & $\begin{array}{l}\text { Restraint } \\
\text { violation } \\
\text { energy }\end{array}$ & $\begin{array}{l}\text { Buried } \\
\text { surface } \\
\text { area }\end{array}$ & Z-score \\
\hline 1BEA & -7.1 & 8 & 0.7 & -81.4 & -243.8 & -4.6 & 1276.9 & 2304.8 & -1.4 \\
\hline $1 \mathrm{EQK}$ & -8 & 7 & 9 & -66.3 & -247.7 & -8.1 & 1158.3 & 2088.9 & -1.7 \\
\hline $1 \mathrm{H} 20$ & -71.3 & 25 & 0.5 & -53.6 & -109.2 & -25 & 291.6 & 1242.3 & -1.3 \\
\hline 1NB1 & -63.5 & 18 & 0.6 & -49.7 & -60.5 & -11.6 & 99 & 1180 & -1.7 \\
\hline 1PJU & 3.3 & 16 & 0.6 & -85.5 & -157 & -6.9 & 1270.4 & 2163.7 & -1.9 \\
\hline 1TIN & -59.6 & 13 & 1 & -55.4 & -326.1 & -9.3 & 702.7 & 2190.8 & -1.5 \\
\hline 2IT7 & -71.4 & 38 & 0.8 & -49.2 & -118.2 & -6.9 & 83.7 & 1279.9 & -2.2 \\
\hline 2PLX & -104.7 & 58 & 0.3 & -63.4 & -194.9 & -11.2 & 89.5 & 1560.7 & -2.2 \\
\hline $5 J 4 Q$ & -47.3 & 29 & 6.2 & -72 & -199.8 & -10.2 & 747.9 & 1985.1 & -1.5 \\
\hline $5 \mathrm{XOZ}$ & 106.5 & 10 & 14.3 & -83.4 & -134 & -1.7 & 2184.4 & 2326.3 & -1.6 \\
\hline $1 \mathrm{JXC}$ & -57 & 50 & 0.3 & -74.3 & -177.5 & -15.7 & 685.9 & 2123.1 & -1.9 \\
\hline
\end{tabular}

of VcTI- PLpro is a stable construct. A steady value of $\mathrm{Rg}$ shows over time is a proof of appropriate conformation of the complex and its stablity (Fig. 5).

\section{Discussion}

COVID-19 has shown to be one of the deadliest viral pandemics in human history. Previously, the world has been affected by two other corona virus epidemic conditions named SARS-CoV and MERS-CoV. The new corona virus, called COVID- 19 by WHO, has $80 \%$ genomic similarity with SARS-CoV, but it has shown to have deadlier outcomes than the other previous epidemics.

Since the emergence of COVID-19, many attempts have been focused on developing preventive agents, such as vaccine and therapeutic reagents against COVID-19. Recently, some advances have been made at developing vaccines against COVID-19, and various types including recombinant multi-epitope vaccines, mRNA vaccines, viral debrides, and engineered recombinant viruses are at their clinical trial.

In spite of developing vaccines, which could be to some degrees effective at preventing the infection, it is necessary 

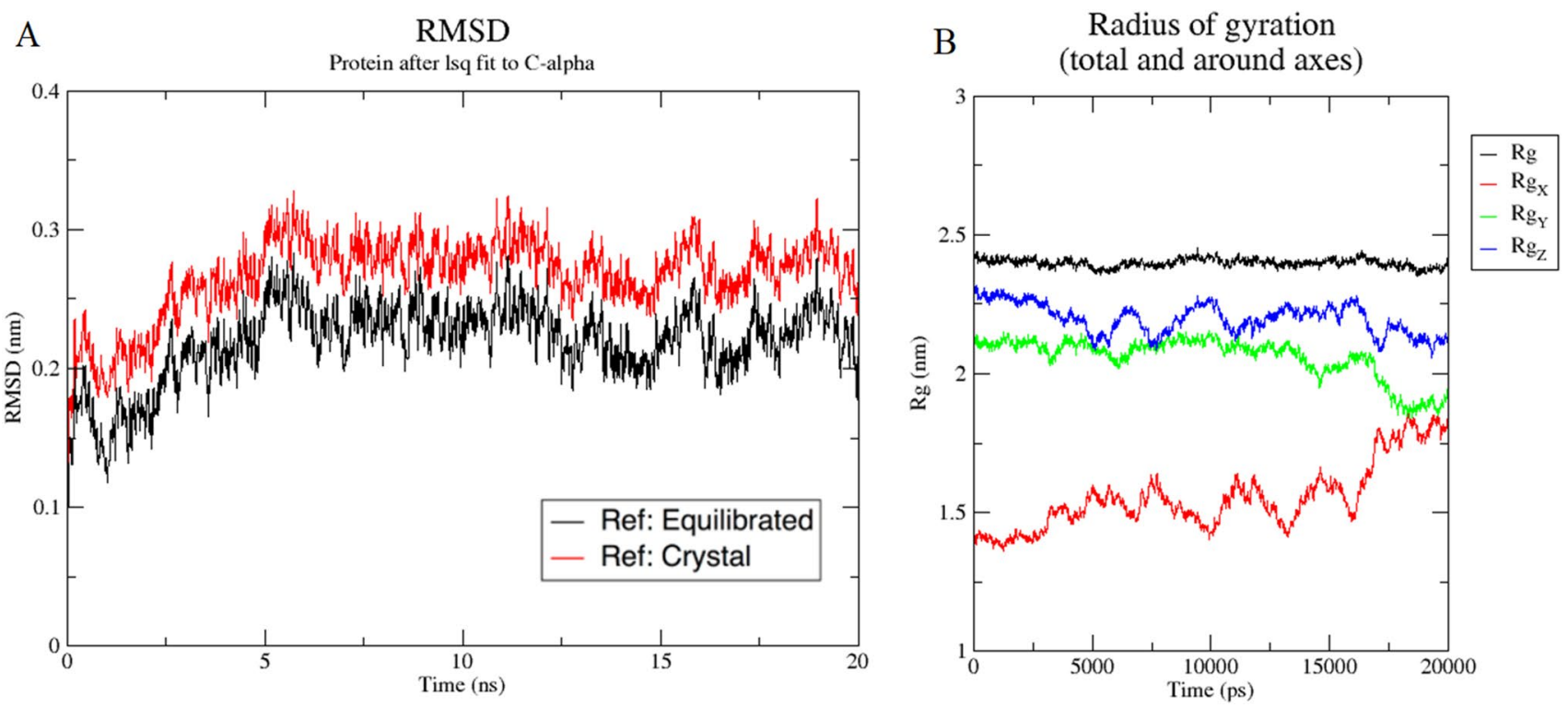

Fig. 5 Molecular dynamics simulation for the VcTI in complex with active site of PLpro enzyme. A. RMSD related to equilibrated (black) and crystal structure (Red) of the complex. B. Radius of gyration of VcTI- PLpro complex (total and around axes)

to find a cure for those who get infected with SARS-CoV-2. Clinical applications of the regular drugs, such as remdesivir has shown some success, but they are not totally effective (Siemieniuk et al. 2020). The use of anti-inflammatory drugs along with antiviral drugs has shown some success (Naveja et al. 2020), but the results were not conclusive enough to be used as a drug for COVID-19 patients. Accordingly, the field of developing anti-viral agents against COVID-19 is still an open area, and scientist with various expertise are still focused on developing an effective therapeutic.

So far, many attempts have been given to find an inhibitor agent against SARS-CoV-2, among which application of natural products has been the subject of many studies. In these studies, in silico assays have been very helpful for screening of the potential therapeutics (Teli et al. 2021) and even designing or optimizing new therapeutics (Behbahani et al. 2021). In this regard, a study by Kurniawan et al. investigated the efficacy of Brazilein sappan wood as a potential inhibitor for spike (S) glycoprotein, papain-like proteinase, and main protease of SARS-CoV-2 by using the platform provided via bioinformatics methods. Their study confirmed the potential of the selected natural products-derived compound for interacting with S protein which was higher than the PLpro and Mpro (Kurniawan and Krihariyani 2021).

One of the interesting in silico studies on screening therapeutic against SARS-CoV-2 was performed by Wong et al., in which they investigated the efficacy of Peptides from Quinoa seed proteins on various targets from SARSCoV-2, including PLpro. They reported that seven peptides from the mentioned source could effectively and stably bind to the viral targets, and none of these peptides were allergenic or toxic to human body, which requires future in vitro and in vivo examinations (Wong et al. 2021a, b). Another study by Srivastavaa et al., investigated the efficacy of phytoconstituents derived from Withania somnifera (a medicinal plant) against SARS-CoV-2 target proteins and human ACE2 through an in silico approach, which showed that Withanolides A and B could effectively bind to spike protein and PLpro components (Srivastava et al. 2020).

Regarding the in silico assays for scrutinizing the potential inhibitors for SARS-CoV-2 papain-like protease inhibitors, a study by Li et al. screened the efficacy of twenty-one antivirals, antifungals and anticancer potential inhibitors against PLpro. Their study showed that among the chosen compounds, Neobavaisoflavone provides the highest binding energy when it is complexed with PLpro enzyme and attached to crucial catalytic triad, ubiquitination and ISGylation amino acids which announced this compound as a potential inhibitor against this viral enzyme (Li et al. 2021).

In the current study, a group of various plant-derived peptides with the potential for suppressing the protease enzymes were selected and their interactions with the two sites of the PLpro were investigated. Molecular docking assay confirmed that among the selected compounds, VcTI from Veronica hederifolia could bind effectively to the both of $\mathrm{Zn}$ site and classic catalytic site of enzyme with the highest docking score and produce effective bonds which could be able to suppress the enzyme activity, therefore presenting a potential therapy for COVID-19.

The complex of PLpro-VcTI in the Zn site of enzyme indicated the highest molecular docking score of -76.1 by HADDOCK tool, which is a comparatively high score for 
binding of ligand to its receptor, with an RMSD of 0.4 , certifying the accuracy of the predicted interactions. For this complex, the Vander Waals energy of $-75.1 \mathrm{kcal} / \mathrm{mole}$ was predicted and $-134.3 \mathrm{kcal} / \mathrm{mole}$ electrostatics energy.

The provided surface area for this complex was 2044.9 $\mathrm{A}^{\mathrm{o}}$ and the $\mathrm{Z}$-score for this predicted complex was -2.2. Moreover, this peptide showed the highest docking score than the other compounds when it was attached to the classical active site of PLpro enzyme by showing the HADDOCK score of -104.7 which was comparatively higher than the other complexes, along with having $-194.9 \mathrm{kcal} /$ mole electrostatics energy. The molecular dynamic assay of this complex verified that VcTI- PLpro complex provided by the molecular docking assay is a stable construct by considering RMSD and Radius of gyration analysis.

This peptide with a single-strand polypeptide, is composed of 26 amino acids could effectively enter the cell membrane and potentially could suppress the activity of this enzyme. The highlighted point about VcTI is that this peptide interacted effectively with the key residues in both $\mathrm{Zn}$ attachment and classic active site of PLpro enzyme. Members of this family of peptides have two antiparallel oriented $\alpha$-helices which are linked together by two disulfide bonds through a specific Cys motif of $\mathrm{C} 1 \mathrm{X} 3 \mathrm{C} 2 \mathrm{XnC} 3 \mathrm{X} 3 \mathrm{C} 4$ (Tam et al. 2015). Having such a construct is helpful for an effective attachment to the both $\mathrm{Zn}$ attachment and the classic active site of PLpro from COVID-19.

Peptides from this family are being reported to suppress trypsin protease enzymes through a classical substrate-like attitude. The binding site of plant-derived PIs usually are at the end of a $\beta$-strand, though the interacting Arg in the prototypic TI peptide VhTI from Veronica hederifolia is placed within a helix-to-helix motif (Bateman 2011). The molecular interactions investigation at the current in silico study also confirmed the importance of interacting Arg residue of this peptide.

It was indicated that regarding the complex at the $\mathrm{Zn}$ attachment site, both critical residues of Cys 192 and Cys 224 were bounded to the Arg 10 of VhTI via hydrogen bonds which provides an effective binding, and the Cys189 residue had non-bound interaction with this Arg10. Moreover, at the catalytic site, the three critical residues were involved in the interaction with the VcTI compound. Arg10 of peptide was again involved in two hydrogen bonds and a salt bridge with Gln161 of the enzyme, and a non-bound interaction with Asn109, showing an important role of this residue at the complex formation.

There is no doubt that the predictions provided by the bioinformatics method could be really helpful to reduce the costs of the laboratory researches and in many cases, these predictions come true and save a considerable time, workforce, and expenses. This study was an in silico research, aiming to develop an effective therapeutic compound against
COVID-19 condition. The results of the bioinformatics investigation suggest that the VcTI compound could potentially suppress the function of this enzyme by attaching two critical sites of this protein, yet future in vitro and in vivo investigations are required to further verify the efficacy of this plant-derived peptide.

\section{Conclusion}

Analyzing the results of molecular interactions study of receptor-ligand complexes between PLpro enzyme of SARS$\mathrm{CoV} 2$ and the selected potential protease inhibitors from different families of plants-derived PIs showed that the complex of VcTI- PLpro provides a more optimal interaction than the other potential ligands, and due to having the comparatively highest scores in both $\mathrm{Zn}$ site and classic active site, this peptide could be a potential inhibitor for PLpro enzyme of COVID-19 that could suppress viral replication, but future in vitro and in vivo investigations are necessary.

Supplementary Information The online version contains supplementary material available at https://doi.org/10.1007/s10989-021-10331-8.

Acknowledgements The authors wish to thank Baqiyatallah University of medical sciences.

Funding None.

Data Availability The data sets used and/or analyzed during the current study are available from the corresponding author on reasonable request.

\section{Declarations}

Conflict of interest None to declare.

Research Involving Human Participants and/or Animals No human or animal was involved in this study.

Informed Consent There was no human participant and consent was not required.

\section{References}

Ahmadi K, Farasat A, Rostamian M, Johari B, Madanchi H (2021) Enfuvirtide, an HIV-1 fusion inhibitor peptide, can act as a potent SARS-CoV-2 fusion inhibitor: an in silico drug repurposing study. J Biomol Struct Dyn. https://doi.org/10.1080/07391102.2021. 1871958

Amin SA, Banerjee S, Ghosh K, Gayen S, Jha T (2021) Protease targeted COVID-19 drug discovery and its challenges: Insight into viral main protease (Mpro) and papain-like protease (PLpro) inhibitors. Bioorg Med Chem 29:115860. https://doi.org/10. 1016/j.bmc.2020.115860 
Báez-Santos YM, St John SE, Mesecar AD (2015) The SARS-coronavirus papain-like protease: structure, function and inhibition by designed antiviral compounds. Antivir Res 115:21-38. https://doi. org/10.1016/j.antiviral.2014.12.015

Barrette-Ng IH, Ng KK-S, Cherney MM, Pearce G, Ghani U, Ryan CA, James MN (2003) Unbound form of tomato inhibitor-II reveals interdomain flexibility and conformational variability in the reactive site loops. J Biol Chem 278(33):31391-31400

Bateman KS, James MNG (2011) Plant protein proteinase inhibitors: structure and mechanism of inhibition. Curr Protein Pept Sci 12(5):341-347

Behbahani M, Mohabatkar H, Hosseini B (2021) In silico design of quadruplex aptamers against the spike protein of SARS-CoV-2. Inform Med Unlocked. https://doi.org/10.1016/j.imu.2021.100757

Behmard E, Soleymani B, Najafi A, Barzegari E (2020) Immunoinformatic design of a COVID-19 subunit vaccine using entire structural immunogenic epitopes of SARS-CoV-2. Sci Rep 10(1):1-12

Behnke CA, Yee VC, Trong IL, Pedersen LC, Stenkamp RE, Kim S-S, Teller DC (1998) Structural determinants of the bifunctional corn Hageman factor inhibitor: X-ray crystal structure at $1.95 \AA$ resolution. Biochemistry 37(44):15277-15288

Bendre AD, Suresh C, Shanmugam D, Ramasamy S (2019) Structural insights into the unique inhibitory mechanism of Kunitz type trypsin inhibitor from Cicer arietinum L. J Biomol Struct Dyn 37(10):2669-2677

Cai M, Gong Y, Kao J, Krishnamoorthi R (1995) Three-dimensional solution structure of Cucurbita maxima trypsin inhibitor-V determined by NMR spectroscopy. Biochemistry 34(15):5201-5211

Conners R, Konarev AV, Forsyth J, Lovegrove A, Marsh J, JosephHorne T, Brady RL (2007) An unusual helix-turn-helix protease inhibitory motif in a novel trypsin inhibitor from seeds of Veronica (Veronica hederifolia L.). J Biol Chem 282(38):27760-27768

Fallah MS, Bayati M, Najafi A, Behmard E, Javad S (2021) Molecular docking investigation of antiviral herbal compounds as potential inhibitors of SARS-CoV-2 spike receptor. Biointerface Res Appl Chem 11:12916-12924

Farady CJ, Craik CS (2010) Mechanisms of macromolecular protease inhibitors. Chembiochem 11(17):2341

Gao X, Qin B, Chen P, Zhu K, Hou P, Wojdyla JA, Cui S (2021) Crystal structure of SARS-CoV-2 papain-like protease. Acta Pharmaceutica Sinica B 11(1):237-245. https://doi.org/10.1016/j.apsb. 2020.08.014

Gharbavi M, Johari B, Rismani E, Mousazadeh N, Taromchi AH, Sharafi A (2020) NANOG decoy Oligodeoxynucleotide-encapsulated noisome nanocarriers: a promising approach to suppress the metastatic properties of U87 human glioblastoma multiforme cells. ACS Chem Neurosci 11(24):4499-4515

Gomes MTR, Oliva ML, Lopes MTP, Salas CE (2011) Plant proteinases and inhibitors: an overview of biological function and pharmacological activity. Curr Protein Pept Sci 12(5):417-436

González C, Neira JL, Ventura S, Bronsoms S, Rico M, Avilés FX (2003) Structure and dynamics of the potato carboxypeptidase inhibitor by $1 \mathrm{H}$ and $15 \mathrm{~N}$ NMR. Proteins Struct Funct Bioinformat 50(3):410-422

Haghighi O (2021) In silico study of the structure and ligand preference of pyruvate kinases from Cyanobacterium Synechocystis sp. PCC 6803. Appl Biochem Biotechnol. https://doi.org/10.1007/ s12010-021-03630-9

Haghighi O, Davaeifar S, Zahiri HS, Maleki H, Noghabi KA (2020) Homology Modeling and Molecular Docking Studies of Glutamate Dehydrogenase (GDH) from Cyanobacterium Synechocystis sp. PCC 6803. Int J Pept Res Therap 26(2):783-793. https://doi. org/10.1007/s10989-019-09886-4

Heitz A, Avrutina O, Le-Nguyen D, Diederichsen U, Hernandez J-F, Gracy J, Chiche L (2008) Knottin cyclization: impact on structure and dynamics. BMC Struct Biol 8(1):1-19
Hellinger R, Gruber CW (2019) Peptide-based protease inhibitors from plants. Drug Discov Today 24(9):1877-1889. https://doi.org/10. 1016/j.drudis.2019.05.026

Hess B, Kutzner C, Van Der Spoel D, Lindahl E (2008) GROMACS 4: algorithms for highly efficient, load-balanced, and scalable molecular simulation. J Chem Theory Comput 4(3):435-447

Ibrahim TM, Ismail MI, Bauer MR, Bekhit AA, Boeckler FM (2020) Supporting SARS-CoV-2 papain-like protease drug discovery: in silico methods and benchmarking. Front Chem. https://doi.org/10. 3389/fchem.2020.592289

Kurniawan E, Krihariyani D (2021) Working goal of Brazilein sappan wood as a candidate for SARS-coV-2 antivirus drug against spike (S) glycoprotein, papain-like proteinase, and main protease: In silico study. J Adv Pharma Technol Res 12(3):298-304. https:// doi.org/10.4103/japtr.JAPTR_222_21

Laskowski RA, Jabłońska J, Pravda L, Vařeková RS, Thornton JM (2018) PDBsum: structural summaries of PDB entries. Protein Sci 27(1):129-134

Li D, Luan J, Zhang L (2021) Molecular docking of potential SARSCoV-2 papain-like protease inhibitors. Biochem Biophys Res Commun 538:72-79. https://doi.org/10.1016/j.bbrc.2020.11.083

Maiti BK (2020) Can papain-like protease inhibitors halt SARS-CoV-2 replication? ACS Pharmacol Transl Sci 3(5):1017-1019. https:// doi.org/10.1021/acsptsci.0c00093

Malik YS, Kumar N, Sircar S, Kaushik R, Bhat S, Dhama K, Ghoshal U (2020) Coronavirus disease pandemic (COVID-19): challenges and a global perspective. Pathogens 9(7):519

Mitra D, Verma D, Mahakur B, Kamboj A, Srivastava R, Gupta S, Panneerselvam P (2021) Molecular docking and simulation studies of natural compounds of Vitex negundo L against papain-like protease (PLpro) of SARS CoV-2 (coronavirus) to conquer the pandemic situation in the world. J Biomol Struct Dyn. https://doi. org/10.1080/07391102.2021.1873185

Mohabatkar H, Behbahani M, Moradi M (2021) A concise in silico prediction report of a potential prion-like domain in SARS-COV-2 Polyprotein. J Microbiol Biotechnol Food Sci. https://doi.org/10. $15414 / j m b f s .4813$

Mohabatkar H, Ebrahimi S, Moradi M (2021b) Using Chou's five-steps rule to classify and predict glutathione S-Transferases with different machine learning algorithms and pseudo amino acid composition. Int J Pept Res Ther 27(1):309-316

Nabati F, Moradi M, Mohabatkar H (2020) In silico analyzing the molecular interactions of plant-derived inhibitors against E6AP, p53, and c-Myc binding sites of HPV type 16 E6 oncoprotein. Mol Biol Res Commun 9(2):71

Nagata K, Kudo N, Abe K, Arai S, Tanokura M (2000) Three-dimensional solution structure of oryzacystatin-I, a cysteine proteinase inhibitor of the rice, Oryza Sativa L. Japonica. Biochemistry 39(48): 14753-14760

Naveja JJ, Madariaga-Mazón A, Flores-Murrieta F, Granados-Montiel J, Maradiaga-Ceceña M, Alaniz VD, Martinez-Mayorga K (2020) Union is strength: antiviral and anti-inflammatory drugs for COVID-19. Drug Discov Today. https://doi.org/10.1016/j. drudis.2020.10.018

Nejat R, Sadr AS (2019) SARS virus papain-like protease: a mysterious weapon. J Biostat Epidemiol 5(4):288-295

Oostenbrink C, Villa A, Mark AE, Van Gunsteren WF (2004) A biomolecular force field based on the free enthalpy of hydration and solvation: the GROMOS force-field parameter sets 53A5 and 53A6. J Comput Chem 25(13):1656-1676

Osipiuk J, Azizi S-A, Dvorkin S, Endres M, Jedrzejczak R, Jones KA, Joachimiak A (2021) Structure of papain-like protease from SARS-CoV-2 and its complexes with non-covalent inhibitors. Nat Commun 12(1):743. https://doi.org/10.1038/s41467-021-21060-3

Pawar SS, Rohane SH (2021) Review on discovery studio: an important tool for molecular docking. Asian J Res Chem 14(1):86-88 
Pettersen EF, Goddard TD, Huang CC, Couch GS, Greenblatt DM, Meng EC, Ferrin TE (2004) UCSF Chimera-a visualization system for exploratory research and analysis. J Comput Chem 25(13):1605-1612

Ram H, Sharma R, Dewan D, Singh A (2020) Insight view on pandemic COVID-19: worldwide perspective with homoeopathic management through repertorial analysis. Homœopathic Link. https://doi.org/10.1055/s-0040-1715865

Rosengren KJ, Daly NL, Plan MR, Waine C, Craik DJ (2003) Twists, knots, and rings in proteins: structural definition of the cyclotide framework. J Biol Chem 278(10):8606-8616

Rut W, Lv Z, Zmudzinski M, Patchett S, Nayak D, Snipas SJ, Drag M (2020) Activity profiling and crystal structures of inhibitor-bound SARS-CoV-2 papain-like protease: a framework for anti-COVID19 drug design. Sci Adv. https://doi.org/10.1126/sciadv.abd4596

Ryu WS (2017) Virus life cycle. Molecular Virology of Human Pathogenic Viruses. Elsevier, Amsterdam

Sargsyan, K., Chen, T., Grauffel, C., \& Lim, C. (2020). Identifying COVID-19 drug-sites susceptible to clinically safe Zn-ejector drugs using evolutionary/physical principles.

Siemieniuk R, Rochwerg B, Agoritsas T, Lamontagne F, Leo Y-S, Macdonald H, Appiah JA (2020) A living WHO guideline on drugs for covid-19. Br Med J 370:m3379

Srivastava A, Siddiqui S, Ahmad R, Mehrotra S, Ahmad B, Srivastava AN (2020) Exploring nature's bounty: identification of Withania somnifera as a promising source of therapeutic agents against COVID-19 by virtual screening and in silico evaluation. J Biomol Struct Dyn. https://doi.org/10.1080/07391102.2020.1835725

Tam JP, Wang S, Wong KH, Tan WL (2015) Antimicrobial peptides from plants. Pharmaceuticals 8(4):711-757

Teli DM, Shah MB, Chhabria MT (2021) In silico screening of natural compounds as potential inhibitors of SARS-CoV-2 main protease and spike RBD: targets for COVID-19. Front Mol Biosci. https:// doi.org/10.3389/fmolb.2020.599079

Tornøe CW, Johansson E, Wahlund P-O (2017) Divergent protein synthesis of Bowman-Birk protease inhibitors, their hydrodynamic behavior and co-crystallization with $\alpha$-chymotrypsin. Synlett 28(15):1901-1906

Valle C, Martin B, Touret F, Shannon A, Canard B, Guillemot JC, Decroly E (2020) Drugs against SARS-CoV-2: what do we know about their mode of action? Rev Med Virol 30(6):1-10

Van Zundert G, Rodrigues J, Trellet M, Schmitz C, Kastritis P, Karaca E, Bonvin A (2016) The HADDOCK2. 2 web server: user-friendly integrative modeling of biomolecular complexes. J Mol Biol 428(4):720-725

Wong F-C, Ong J-H, Chai T-T (2021) SARS-CoV-2 spike protein-, main protease-and papain-like-protease-targeting peptides from seed proteins following gastrointestinal digestion: an in silico study. Phytomed Plus 1(1):100016

Wong F-C, Ong J-H, Kumar DT, Chai T-T (2021b) In silico identification of multi-target anti-SARS-CoV-2 peptides from quinoa seed proteins. Int J Pept Res Ther 27(3):1837-1847. https://doi.org/10. 1007/s10989-021-10214-y

Zhao Q, Chae YK, Markley JL (2002) NMR solution structure of ATTp, an Arabidopsis thaliana trypsin inhibitor. Biochemistry 41(41):12284-12296

Publisher's Note Springer Nature remains neutral with regard to jurisdictional claims in published maps and institutional affiliations. 